OPEN ACCESS

Edited by:

Girolamo Pelaia,

University of Catanzaro, Italy

Reviewed by:

Bertrand De Meulder,

European Institute for Systems

Biology and Medicine (EISBM), France

Feng-Ming Yang,

Taipei Medical University, Taiwan

*Correspondence:

Heung-Woo Park

guinea71@snu.ac.kr

orcid.org/0000-0002-6970-3228

Specialty section: This article was submitted to

Pulmonary Medicine,

a section of the journal

Frontiers in Medicine

Received: 13 January 2021 Accepted: 25 February 2021

Published: 18 March 2021

Citation:

Kim B-K, Lee H-S, Lee S-Y and Park H-W (2021) Different Biological Pathways Between Good and Poor Inhaled Corticosteroid Responses in

Asthma. Front. Med. 8:652824.

doi: 10.3389/fmed.2021.652824

\section{Different Biological Pathways Between Good and Poor Inhaled Corticosteroid Responses in Asthma}

\author{
Byung-Keun Kim ${ }^{1}$, Hyun-Seung Lee ${ }^{2}$, Suh-Young Lee ${ }^{3}$ and Heung-Woo Park ${ }^{2,3,4 *}$ \\ ${ }^{1}$ Department of Internal Medicine, Korea University College of Medicine, Seoul, South Korea, ${ }^{2}$ Institute of Allergy and Clinical \\ Immunology, Seoul National University Medical Research Center, Seoul, South Korea, ${ }^{3}$ Department of Internal Medicine, \\ Seoul National University Hospital, Seoul, South Korea, ${ }^{4}$ Department of Internal Medicine, Seoul National University College \\ of Medicine, Seoul, South Korea
}

Gene regulatory networks address how transcription factors (TFs) and their regulatory roles in gene expression determine the responsiveness to anti-asthma therapy. The purpose of this study was to assess gene regulatory networks of adult patients with asthma who showed good or poor lung function improvements in response to inhaled corticosteroids (ICSs). A total of 47 patients with asthma were recruited and classified as good responders (GRs) and poor responders (PRs) based on their responses to ICSs. Genome-wide gene expression was measured using peripheral blood mononuclear cells obtained in a stable state. We used Passing Attributes between Networks for Data Assimilations to construct the gene regulatory networks associated with GRs and PRs to ICSs. We identified the top-10 TFs that showed large differences in high-confidence edges between the GR and PR aggregate networks. These top-10 TFs and their differentially-connected genes in the PR and GR aggregate networks were significantly enriched in distinct biological pathways, such as TGF- $\beta$ signaling, cell cycle, and IL-4 and IL-13 signaling pathways. We identified multiple TFs and related biological pathways influencing ICS responses in asthma. Our results provide potential targets to overcome insensitivity to corticosteroids in patients with asthma.

Keywords: asthma, gene expression, gene regulatory networks, inhaled corticosteroid, transcription factor, pharmacogenomics, blood

\section{INTRODUCTION}

Blood contains many cells involved in immune responses, which explains why blood cell transcriptomics has been used for the study of asthma, an immune-mediated disease. For instance, it was reported that $M K P-1$ and $I L-8$ gene expression in peripheral blood mononuclear cells (PBMCs) of patients with asthma was useful in predicting clinical response to corticosteroids (1).

Recent transcriptomic studies have focused on the biological systems that are organized by various molecular entities such as genes, proteins and metabolites as well as the interactions between them. These systems can be visualized as networks, also interchangeably recognized as acyclic graphs, in which components (e.g., genes, proteins, or metabolites) are nodes that are connected by edges (relationships between nodes) (2). One good example is a gene co-expression network based on the similar, or correlated, gene expression patterns (3). 
However, correlation does not necessarily imply causation. Gene regulatory networks attempt to identify the influencing patterns of transcription factors (TFs) on gene expression in a mechanistic fashion (4). As reviewed before, the activation or repression of different TFs and their regulatory roles in gene expression may determine the responsiveness to antiasthma therapy, particularly to anti-inflammatory drugs (5). Qiu et al. found that TFs differentially affected gene expression in lymphoblastoid cell lines from children with asthma that included good and poor responders to inhaled corticosteroid (ICS) treatment by applying gene regulatory networks (6).

The purpose of this study was to assess gene regulatory networks of adult patients with asthma who showed good or poor lung function improvements in response to ICSs. To do this, we began by analyzing genome-wide gene expression levels in PBMCs from adult patients with asthma. Following this, we explored whether gene regulatory networks showed good or poor responder-specific regulatory patterns using the Passing Attributes between Networks for Data Assimilation (PANDA) algorithm. PANDA models information flow through networks under the assumption that both "transmitters" and "receivers" play active roles in modulating regulatory processes (7).

\section{MATERIALS AND METHODS}

This study was approved by the Institutional Review Board of the corresponding institution (H-1408-051-601 and 2019AN0240) and informed consent was obtained from all study participants.

\section{Study Populations}

We retrospectively reviewed the medical records of two institutes (Seoul National University Hospital and Korea University Anam Hospital, Seoul, Republic of Korea) and selected adult patients with asthma eligible for our study. The diagnosis of asthma was confirmed when forced expiratory volume in $1 \mathrm{~s}$ (FEV1) showed more than $12 \%$ (and $200 \mathrm{~mL}$ ) increase after initiation of treatment. After diagnosis, all of patients were treated with medium dose ICSs (8) and regularly followed up; the pulmonary function measurement was performed every 4 weeks. Current or former smokers were excluded. We explained our study to eligible patients with asthma identified from medical records and enrolled them if they agreed to participate. We defined poor responders (PRs) or good responder (GRs) to ICSs, as patients who had less or more than $12 \%$ improvement in FEV1 compared to baseline values at 4 weeks after initiation of treatment, respectively (1). PRs eventually achieved more than $12 \%$ improvement in FEV1 responding to ICSs, but it took longer than 4 weeks. The overall study design is presented in Figure $\mathbf{1}$.

\section{Gene Expression Arrays}

Blood for gene expression analysis was drawn at a stable state, that is, no changes in anti-asthma medications and no acute exacerbations (short-term oral prednisone burst, unexpected

Abbreviations: EES, Edge Enrichment Score; FEV1, Forced expiratory volume in 1 second; GR, Good responder; ICS, Inhaled corticosteroid; PANDA, Passing Attributes between Networks for Data Assimilation; PBMCs, Peripheral blood mononuclear cells; PR, Poor responder; TF, Transcription factor.

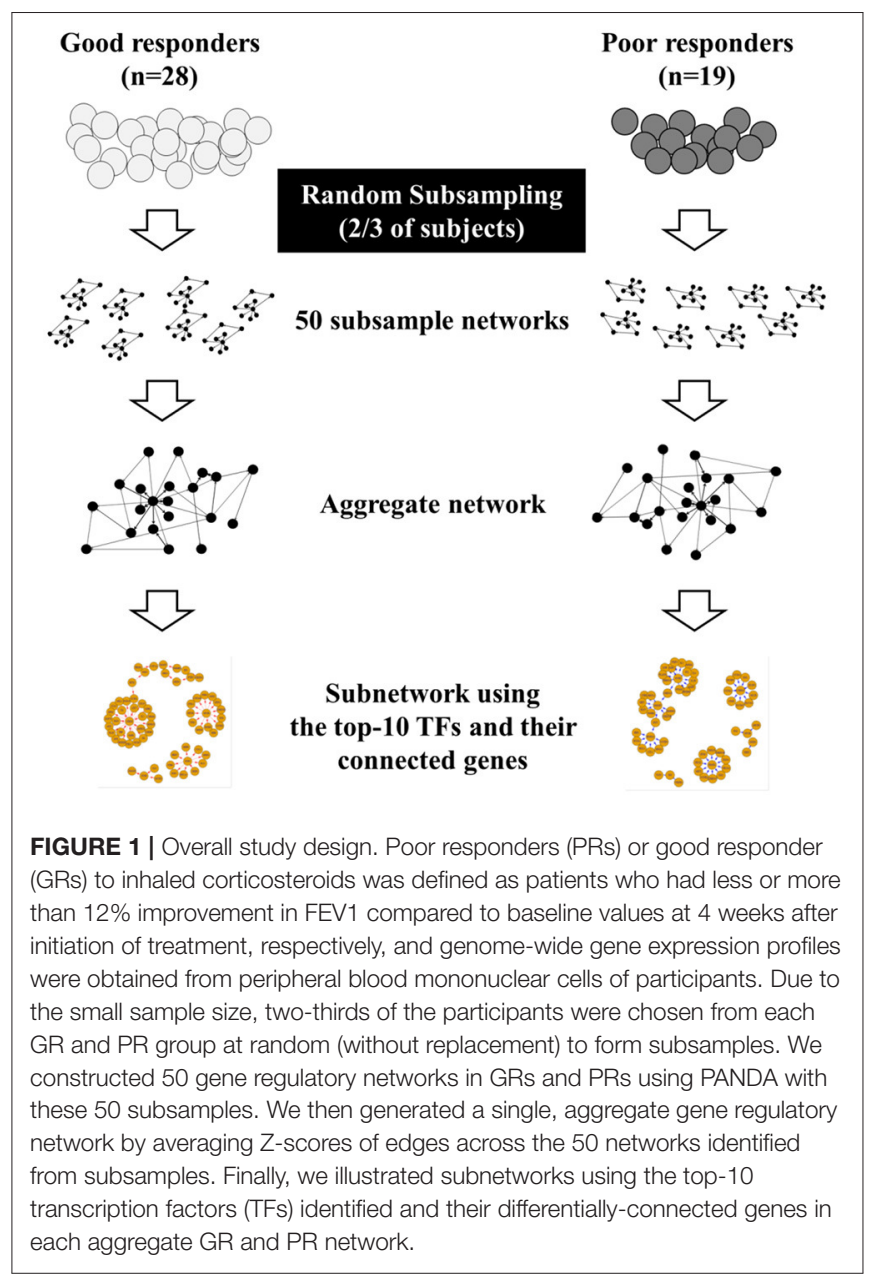

clinic visit, and emergency room visit or hospitalization due to asthma symptom aggravation) within 4 weeks prior to blood sampling. PBMCs were isolated and genome-wide gene expression levels were measured using the Affymetrix GeneChip Human Gene 2.0 ST (Affymetrix, Santa Clara, CA, USA). We removed probes with bad chromosome annotations and probes in the $\mathrm{X}$ or $\mathrm{Y}$ chromosome. We then performed variancestabilizing transformation and quantile-normalization to reduce technical noises and to make the distribution of expression level for each array closer to a normal distribution.

\section{Network Analysis}

The analysis was performed with $\mathrm{R}$ version 4.0.2 (www.r-project.org). We performed PANDA analysis on gene expression profiles from GRs and PRs using the R package "pandaR" (9). network. To seed the PANDA algorithm, we used a mapping between TF motifs and target genes from the TRRUST database (10). This mapping file consists of 8,444 regulatory interactions for 800 TFs and 2,521 target genes. There are 796 TFs in both our gene expression data and the mapping file. These TFs correspond to 9,392 pairs of (TF, gene) and correspond to 2,490 genes in our expression data. 
To minimize the effect of outliers in our networks that were built on a smaller sample size, two-thirds of the participants were chosen from each GR and PR group at random (without replacement) to form subsamples. These 50 subsamples were used to construct 50 gene regulatory networks in GRs and PRs. PANDA reports the probability that a connection (edge) exists between a TF and gene in an estimated network as a Z-score (7). We generated a single, aggregate gene regulatory network by averaging $\mathrm{Z}$-scores of edges across the 50 networks identified from subsamples, as described elsewhere (11). We then selected high-confidence edges that had an average edge $\mathrm{Z}$-score $>0$ in the aggregate GR or PR networks. These edges can be interpreted as edges that are most likely to exist in each aggregate network.

To quantify differences in high-confidence edges, we calculated an edge enrichment score (EES) (11): $E E S_{i}=$ $\log _{2}\left[\left(k^{\mathrm{g}} / k_{i}^{\mathrm{p}}{ }_{i}\right) /\left(N^{\mathrm{g}} / N^{\mathrm{p}}\right)\right]$ where $k^{\mathrm{g}}{ }_{i}$ and $k^{\mathrm{p}}{ }_{i}$ are the (out-degree) number of high-confidence edges for TF $i$ in the aggregate GR and PR networks, respectively, and $N^{\mathrm{g}}$ and $N^{\mathrm{P}}$ are the total number of high-confidence edges in each network. Note that the EES is positive for edge-enrichment from a particular TF in the aggregate GR network, and negative for edge-enrichment from a particular $\mathrm{TF}$ in the aggregate PR network.

\section{Gene Set Enrichment Analysis}

Based on EES, we selected the top-10 TFs from aggregate networks (5 of the highest ones and 5 of the lowest ones). We then identified genes connected to these 10 TFs differentially in the aggregate GR and PR networks by selecting genes whose differences in high-confidence edge Z-scores were $>0.75$. This means that these genes have at least a $75 \%$ chance of existing and being different in each aggregate network. As we assumed that these 10 TFs and their differentially-connected genes were the main drivers in each aggregate network, we constructed GR and PR subnetworks using them. To assign biological meaning to interpretability of each subnetwork, we performed pathway overrepresentation analysis (gene set enrichment analysis) of individual TF and its target genes in the GR and PR subnetworks using "g:Profiler" (database version: e100_eg47_p14_7733820) (12). g:Profiler (https://biit.cs.ut.ee/ gprofiler/) provides an adjusted $P$-value calculated in a manner that accounts for the hierarchical relationships among the tested gene sets. g:Profiler utilizes 3 types of biological pathways; KEGG, Reactome, and WikiPathways. As Reactome provides more and diverse signaling pathways including immunological, developmental and kinase, signaling pathways, the drugor target-based, stress activated, or lipid-mediated signaling pathways compared to the other databases (13), we selected Reactome database.

\section{RESULTS}

A total of 47 adult patients with asthma (28 GRs and 19 PRs) were enrolled. Table 1 summarizes their baseline characteristics. There were no significant differences between GRs and PRs for age, sex, atopy, blood eosinophil counts, or pulmonary functions at baseline (before initiation of treatment). GRs showed
TABLE 1 | Characteristics of enrolled patients with asthma.

\begin{tabular}{lccc}
\hline & Good responder & Poor responder & P-value \\
& $\boldsymbol{n}=\mathbf{2 8}$ & $\boldsymbol{n}=\mathbf{1 9}$ & \\
\hline Age (year) & $51.9(13.8)$ & $52.8(15.6)$ & 0.83 \\
Male & $9(32.1 \%)$ & $8(42.1 \%)$ & 0.75 \\
Atopy & $15(53.6 \%)$ & $8(42.1 \%)$ & 0.64 \\
Blood eosinophil $(/ \mu L)$ & $541.7(220.4)$ & $605.3(349.6)$ & 0.48 \\
FEV1 (ml) & $1,897.1(501.3)$ & $2,323.1(987.6)$ & 0.057 \\
FEV1 predicted (\%) & $67.5(15.2)$ & $71.9(19.2)$ & 0.092 \\
FVC (mL) & $2,770.3(684.6)$ & $3,178.4(1,115.1)$ & 0.12 \\
FVC predicted (\%) & $79.1(13.5)$ & $86.2(15.8)$ & 0.11 \\
FEV1/FVC ratio (\%) & $66.5(11.5)$ & $71.9(10.3)$ & 0.11 \\
FEV1 increase (mL) & $694.3(410.9)$ & $78.4(172.5)$ & $1.82 \times 10^{-7}$ \\
FEV1 increase (\%) & $45.5(42.7)$ & $3.7(8.1)$ & $1.24 \times 10^{-4}$ \\
\hline
\end{tabular}

FEV1, Forced expiratory volume in 1 second; FVC, Forced vital capacity.

${ }^{a}$ Differences between FEV1 measured at baseline and FEV1 measured at 4 weeks after initiation of treatment. Data are presented as "mean (standard deviation)" except for male and atopy which are presented as "number (\%)."

TABLE 2 | Top-10 transcription factors identified from aggregate networks based on edge enrichment scores.

\begin{tabular}{lcccc}
\hline TF & $\boldsymbol{n E d g e ( G R )}$ & $\boldsymbol{n E d g e ( P R )}$ & $\boldsymbol{n \text { Diff }}$ & Log $_{\mathbf{2}}$ (EES) \\
\hline MXD1 & 582 & 185 & 397 & 1.653 \\
NFYB & 430 & 142 & 288 & 1.598 \\
E2F6 & 621 & 212 & 409 & 1.551 \\
CREM & 828 & 290 & 538 & 1.514 \\
RFX2 & 590 & 209 & 381 & 1.497 \\
ID3 & 235 & 662 & -427 & -1.494 \\
HOXA1 & 279 & 818 & -539 & -1.552 \\
JUNB & 268 & 823 & -555 & -1.619 \\
PROX1 & 205 & 678 & -473 & -1.726 \\
SMAD7 & 57 & 323 & -266 & -2.503 \\
\hline
\end{tabular}

TF, transcription factor; $n$, number; diff, difference; EES, edge enrichment score; GR, good responder; PR, poor responder.

significant improvement in FEV1 compared to PRs at 4 weeks after initiation of treatment [694.3 $\pm 410.9 \mathrm{~mL}$ (GR) vs. 78.4 $\pm 172.5 \mathrm{~mL}(\mathrm{PR}), P=1.82 \times 10^{-7}$ ), as expected from the definition of 2 groups. Using PANDA, we created aggregate GR and PR networks and identified the top-10 TFs based on EES. Table 2 lists top-5 TFs of those with the highest EES and top-5 TFs of those with the lowest EES. MXD1 has the highest EES, which means that high-confidence edges connecting MXD1 and genes are most greatly enriched in the aggregate GR network. Meanwhile SMAD7 with the lowest EES is positioned in the opposite end. Table 2 also shows absolute numbers of high-confidence edges connecting top-10 TFs and genes in each aggregate GR or PR network and net differences between two aggregate networks. It was difficult to visualize the differential connectivity of TFs and connected genes in the aggregate GR and PR networks, if all TF-gene connections were considered. Hence, we illustrated subnetworks 

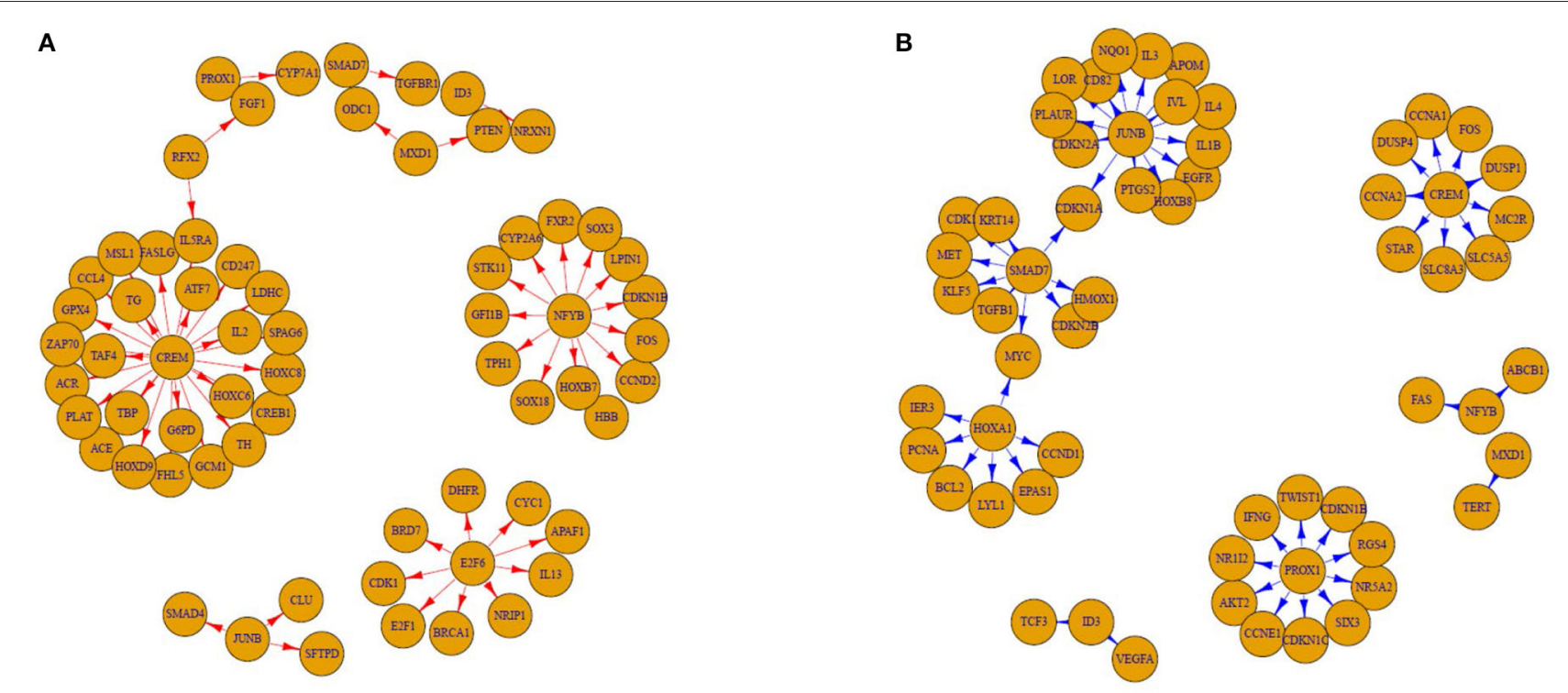

FIGURE 2 | Good (GR) and poor responder (PR) subnetwork made by the top-10 transcription factors and their differentially-connected genes. (A) GR and (B) PR. Based on edge enrichment score, we selected the top-10 TFs from aggregate networks (5 of the highest ones and 5 of the lowest ones). We then identified genes connected to these 10 TFs differentially in the aggregate GR and PR networks and illustrated subnetworks using them. The edges are directed from TFs to their targeting genes whose differences in high-confidence edge Z-scores are $>0.75$. This means that these genes have at least a $75 \%$ chance of existing and being different in each aggregate network.

using the top-10 TFs identified and their differentially-connected genes in each aggregate GR and PR network. Figure 2 shows the differential connectivity of top-10 TFs to genes between aggregate GR and PR network. E2F6 and RFX2 are connected to genes with high-confidence in aggregate GR network only, whereas HOXA1 is connected to genes in aggregate PR network only. The other 7 TFs are differentially connected to genes between aggregate GR and PR networks. The name of genes connected to the top-10 TFs in each aggregate network is listed in Table 3. Table 4 summarizes identified biological pathways in which TF and its connected genes in each aggregate network are enriched with adjusted $\mathrm{P}$ values less than 0.001. For example, E2F6 and its connected genes in aggregate GR network (APAF1, BRCA1, BRD7, CDK1, CYC1, DHFR, E2F1, IL13, and NRIP1) are significantly enriched in G1/S-Specific Transcription, Transcriptional Regulation by E2F6, G1/S Transition, Mitotic G1 phase and G1/S transition, and Transcriptional Regulation by TP53 pathways. The pathways that were identified helped us understand differences in regulatory control driven by Top10 TFs between GR and PR. In the GR subnetwork, E2F6, and NFYB supposedly play important roles by regulating cell cycle-related and FOXO-mediated transcription pathways, respectively. Meanwhile, CREM, PROX1, and SMAD7 are crucial in the PR subnetwork controlling cell cycle-related, immunemediated and TGF- $\beta$ signaling pathways. Interestingly, JUNB is engaged in both GR and PR subnetworks. However, it differentially regulates biological pathways (TGF- $\beta$ vs. IL-4 and IL-13 signaling pathways). The top- 10 TFs identified in this study are not differentially expressed between GR and PR groups (data not shown).

\section{DISCUSSION}

In this study, we constructed gene regulatory networks associated with good or poor response to ICSs using gene expression profiles of PBMCs from 47 adult patients with asthma. We identified the top-10 TFs that showed large differences in EES in the aggregate GR and PR networks. We also identified subnetworks made by top-10 TFs and their differentially-connected genes in each aggregate GR and PR network. In addition, these TFs and genes were enriched in distinctly different biological pathways in GRs and PRs. Based on our results, we summarize that TGF$\beta$ signaling, cell cycle related, and IL-4 and IL-13 signaling pathways are important in determining responses to ICSs in patients with asthma.

Interestingly, the top-10 TFs and their differentiallyconnected genes in our regulatory networks showed no significant differences in expression between GRs and PRs (data not shown). It is possible that multiple TFs compete for the same binding site of a target gene, but which one primarily regulates that gene is dependent on the drug response phenotype. Gene regulatory networks provide us an opportunity to model biological processes as information flowing between genes and the potential to identify the underlying causes of the drug response that cannot be captured by differential gene expression networks. PANDA constructs networks based on differential connectivity by comparing differential expression of the TFs. Therefore, the same sets of TFs may regulate different sets of downstream genes between GRs and PRs, as we observed with JUNB in our analysis. Our results indicated that responses between good and poor responders to a certain drug does not 
TABLE 3 | Top-10 transcription factors and their differentially-connected genes in the aggregate good and poor responder network.

\begin{tabular}{|c|c|}
\hline Transcription factor & Connected genes \\
\hline \multicolumn{2}{|l|}{ Good responder only } \\
\hline E2F6 & APAF1; BRCA1; BRD7; CDK1; CYC1; DHFR; E2F1; IL13; NRIP1 \\
\hline RFX2 & FGF1; IL5RA \\
\hline \multicolumn{2}{|l|}{ Poor responder only } \\
\hline HOXA1 & BCL2; CCND1; EPAS1; IER3; LYL1; MYC; PCNA \\
\hline \multicolumn{2}{|l|}{ Common } \\
\hline \multirow[t]{2}{*}{ CREM } & $\begin{array}{l}\text { [Good responder] ACE; ACR; ATF7; CCL4; CD247; CREB1; FASLG; FHL5; G6PD; GCM1; GPX4; HOXC6; HOXC8; HOXD9; IL2; } \\
\text { IL5RA; LDHC; MSL1; PLAT; SPAG6; TAF4; TBP; TBP; TG; TH; ZAP70 }\end{array}$ \\
\hline & [Poor responder] CCNA1; CCNA2; DUSP1; DUSP4; FOS; MC2R; SLC5A5; SLC8A3; STAR \\
\hline \multirow[t]{2}{*}{ ID3 } & [Good responder] NRXN1 \\
\hline & [Poor responder] TCF3; VEGFA \\
\hline \multirow[t]{2}{*}{ JUNB } & [Good responder] CLU; SMAD4; SFTPD \\
\hline & [Poor responder] APOM; CD82; CDKN1A; CDKN2A; EGFR; HOXB8; IL1B; IL3; IL4; IVL; LOR; NQO1; PLAUR; PTGS2 \\
\hline \multirow[t]{2}{*}{ MXD1 } & [Good responder] ODC1; PTEN \\
\hline & [Poor responder] TERT \\
\hline \multirow[t]{2}{*}{ NFYB } & [Good responder] CCND2; CDKN1B; CYP2A6; FOS; FXR2; GFI1B; HBB; HOXB1; LPIN1; SOX18; SOX3; STK11; TPH1 \\
\hline & [Poor responder] ABCB1; FAS \\
\hline \multirow[t]{2}{*}{ PROX1 } & [Good responder] CYP7A1 \\
\hline & [Poor responder] AKT2; CCNE1; CDKN1B; CDKN1C; IFNG; NR1I2; NR5A2; RGS4; SIX3; TWIST1 \\
\hline \multirow[t]{2}{*}{ SMAD7 } & [Good responder] TGFBR1 \\
\hline & [Poor responder] CDK1; CDKN1A; CDKN2B; HMOX1; KLF5; KRT14; MET; MYC; TGFB1 \\
\hline
\end{tabular}

necessarily emanate from differential gene expression networks but may instead be from regulatory gene expression networks.

SMAD7 had the lowest EES, which suggests that it may play an important role in the aggregate PR network. Binding of TGF- $\beta$ to its receptor triggers phosphorylation of SMAD2 and SMAD3 and phosphorylated SMAD2/3 proteins heterodimerize with SMAD4 to generate a complex that moves to the nucleus, where it regulates the expression of target genes (canonical TGF- $\beta$ signaling) (14). This TGF- $\beta$-associated SMAD signaling is tightly controlled by SMAD7, another intracellular SMAD protein (15). Thus, SMAD 7 acts as a negative regulator of the canonical TGF- $\beta$ signaling pathway. Previously, it was reported that TGF- $\beta$ impairs therapeutic responses to corticosteroids in chronic airway diseases and the non-canonical signaling pathway is important in this process $(16,17)$. However, these reports were based on experiments using lung epithelial cells. TGF- $\beta$ regulates pathologic $\mathrm{CD} 4+\mathrm{T}$ cell responses by directly suppressing $\mathrm{T}$ bet and GATA-3 expression and by downregulating both Th1 and Th2 cell differentiation $(18,19)$. In addition, TGF- $\beta$ can promote the induction of regulatory $\mathrm{T}$ cells (20). For dendritic cells, TGF- $\beta$ can downregulate the antigen-presenting function and expression of co-stimulatory molecules in vitro (21). Mice over-expressing Smad7 in $\mathrm{T}$ cells develop severe intestinal inflammation in various experimental models (22). In this study, we examined gene expression in PBMCs from adult patients with asthma, contrary to previous studies using lung epithelial cells. This would explain why Smad7 influence increases in the aggregate PR network in our study. In addition, we observed that JUNB is confidently connected with SMAD4 in the GR subnetwork (Figure 2A). A previous report showed that JUNB is a critical activator protein component mediating TGF- $\beta$ signaling in human breast epithelium (23). Taken together, increased TGF- $\beta$ signaling in blood cells may confer good response to ICSs in asthma. Although it was recently reported that knockdown of SMAD7 with a specific antisense oligonucleotide that restores endogenous TGF- $\beta$ activity is not effective for patients with steroid-resistant/dependent Crohn disease (24), a SMAD7-targeting approach is worthy of being searched to treat patients with insensitivity to corticosteroids in asthma.

Corticosteroid possesses an anti-inflammatory action and inhibits various inflammatory chemokines and cytokines, including IL-4 and IL-13 (25). Unexpectedly, we found that JUNB and its related genes in the PR subnetwork were significantly enriched in the IL-4 and IL-13 signaling pathway. Moreover, we observed that E2F6 is connected to IL-13 in the GR subnetwork. Bruhn et al. have suggested an inhibitory role for E2F6 in the regulation of IL-13 and allergy based on gene expression analysis of CD4+ T cells (26). As participants in this study were treated with medium dose ICS, it was possible that the amount of corticosteroids was not enough to suppress IL-4 and IL-13 signaling pathway entirely. Another possible explanation is that corticosteroids in their conventional doses are not sufficient to suppress IL-4 and IL-13 signaling pathway completely in some patients. For example, the 1-week course of prednisone treatment did not show significant changes in bronchoalveolar lavage cells expressing IL-4 and IL-13 mRNA in patients with asthma who were recognized as PRs to corticosteroids (27). In this sense, the role of dupilumab, a monoclonal antibody blocking IL-4 and IL-13 signaling pathway by inhibiting IL$4 \mathrm{R}$ alpha, is promising for the management of patients with 
TABLE 4 | Reactome pathways significantly enriched by the top-10 transcription factors and their differentially-connected genes in the good and poor responder subnetworks.

\begin{tabular}{|c|c|c|c|c|}
\hline \multirow[t]{2}{*}{ TF } & \multicolumn{2}{|l|}{ Good responder } & \multicolumn{2}{|l|}{ Poor responder } \\
\hline & Pathway name & $P$-value* & Pathway name & $P$-value* \\
\hline \multirow[t]{5}{*}{ E2F6 } & G1/S-Specific Transcription & 2.02E-06 & None & \\
\hline & Transcriptional Regulation by E2F6 & 4.57E-06 & & \\
\hline & G1/S Transition & 0.001104 & & \\
\hline & Mitotic G1 phase and G1/S transition & 0.001794 & & \\
\hline & Transcriptional Regulation by TP53 & 0.002374 & & \\
\hline \multirow[t]{2}{*}{ CREM } & None & & $\begin{array}{l}\text { Phosphorylation of proteins involved in the G2/M } \\
\text { transition by Cyclin A:Cdc2 complexes }\end{array}$ & 0.000465 \\
\hline & & & G2 Phase & 0.001549 \\
\hline NFYB & FOXO-mediated transcription & 0.006694 & None & \\
\hline \multirow[t]{3}{*}{ JUNB } & $\begin{array}{l}\text { SMAD2/SMAD3:SMAD4 heterotrimer regulates } \\
\text { transcription }\end{array}$ & 0.007895 & Interleukin-4 and interleukin-13 signaling & 0.000039 \\
\hline & & & Signaling by interleukins & 0.002486 \\
\hline & & & Cytokine signaling in immune system & 0.005848 \\
\hline \multirow[t]{6}{*}{ PROX1 } & None & & Mitotic G1 phase and G1/S transition & 0.000534 \\
\hline & & & PTK6 regulates cell cycle & 0.001627 \\
\hline & & & Cyclin E associated events during G1/S transition & 0.005497 \\
\hline & & & Cyclin A:Cdk2-associated events at S phase entry & 0.005905 \\
\hline & & & AKT phosphorylates targets in the cytosol & 0.009838 \\
\hline & & & $\begin{array}{l}\text { TP53 regulates transcription of genes involved in G1 cell } \\
\text { cycle arrest }\end{array}$ & 0.009838 \\
\hline \multirow[t]{5}{*}{ SMAD7 } & None & & $\begin{array}{l}\text { SMAD2/SMAD3:SMAD4 heterotrimer regulates } \\
\text { transcription }\end{array}$ & 0.000503 \\
\hline & & & Mitotic G1 phase and G1/S transition & 0.001057 \\
\hline & & & $\begin{array}{l}\text { Transcriptional activity of SMAD2/SMAD3:SMAD4 } \\
\text { heterotrimer }\end{array}$ & 0.001335 \\
\hline & & & $\begin{array}{l}\text { TFAP2 (AP-2) family regulates transcription of cell cycle } \\
\text { factors }\end{array}$ & 0.001549 \\
\hline & & & Signaling by TGF-beta receptor complex & 0.005696 \\
\hline
\end{tabular}

*adjusted $P$-values.

asthma with reduced response to corticosteroids, as reviewed recently (28). An interesting finding is that the cell cycle (G1/S transition) related pathways are significantly enriched in both GR and PR subnetworks. The accurate transition from G1 (Gap 1) phase of the cell cycle to $S$ (Synthesis) phase is crucial for the control of eukaryotic cell proliferation (29). Since long time ago, we have known that dexamethasone induces irreversible G1 arrest and death of a human lymphoid cell line (30). An arrest of cell cycle progression in the G1/S phase also induced apoptosis of human eosinophils from patients with asthma (31). All these together imply a potential role of the G1/S transition in blood cells in response to corticosteroids. We found that E2F6 and its differentially-connected genes in the GR subnetwork and CREM and PROX1 and their differentiallyconnected genes in the PR subnetwork are enriched in the G1/S transition related pathways. E2F6 functions as a repressor of E2F-dependent transcription during $\mathrm{S}$ phase and thus is presumed to be a cell cycle transcriptional repressor (32). Meanwhile, CREM is implicated in the stimulation of cyclin A transcription at G1/S (33). PROX1, has conflicting roles in cell cycle regulation. PROX1 induces cell cycle arrest in liver hepatocellular carcinoma cells (34), but paradoxically increases proliferation in fetal hepatoblasts (35). These findings suggest that PROX1 regulates the cell cycle in a cell-type-dependent manner. Taken together, cell cycle arrest at G1/S may help to avoid steroid resistance. In addition, it was reported that dexamethasone can stimulate the G1/S transition in human airway fibroblasts in asthma, which may result in airway remodeling (36). In a study reported by Goleva E et al. (1), significantly more dexamethasone was required to suppress in vitro $\mathrm{T}$ cell proliferation in PBMC from steroid resistant than steroid sensitive asthmatics. Taken together, an intrinsic property related with PBMC proliferation in asthmatics may determine the susceptibility for corticosteroid treatment and thus a new approach focused on the G1/S transition is worthy of being investigated to overcome corticosteroid insensitivity.

RFX2 and HOXA1 may also play an important role in determining response to ICSs. Probably, the number of targeted genes with difference in high-confidence edge Z-scores greater than 0.75 between GR and PR groups is too small to be captured as a specific biologic pathway. As gene expression changes over time, the sampling time of PBMCs in this study may not be the 
exact time representing the whole picture of RFX2- or HOXA1related mechanisms.

A small number of participants in this study is a potential limitation. To minimize heterogeneity because of this, we constructed regulatory networks using random subsampling of participants and averaged these networks to identify aggregate GR and PR networks. By doing this, we removed the effect of changes in gene expression that are specific to only one individual (outliers) and focus on changes that are most likely a result of corticosteroid responses. We assumed that TFs with greater differences in high-confidence edges were the main drivers in each aggregate network. Moreover, it was difficult to visualize all the differential connectivity of TFs and their connected genes in the aggregate networks. For these reasons, we selected only the top-10 TFs with a quantified method (EES) focusing on the largescale changes in edge numbers between two aggregate networks. However, it is possible that other TFs and their related genes excluded from our analysis would have their potential roles in determining ICS responses. Despite taking these precautions, we recognize that future studies are needed for the functional validation of our networks.

In addition, the statistical power and sample size should be considered before generalizing our observations. The performance of gene regulatory network inference algorithms with a genome-wide scale depends on the sample size. It is generally considered that the larger the sample size, the better the gene network inference performance. However, there has not been adequate information on determining the sample size for optimal performance of gene regulatory network inference. In one study using a pseudo gene regulatory network with 6 nodes which is generated from gene-gene associations based on the coefficient of intrinsic dependence, the false networks only appears $\leq 5$ times in 100 simulations for the sample size $=25,50$, and 100 (37). In other study based on the real world gene expression data sets, it was reported that the sample size around 64 is sufficient to obtain acceptable performance of the information-theory-based gene regulatory network inference algorithms (38). We cannot directly apply previous observations to ours, as inference algorithms are different with that of PANDA. However, considering previous reports, we may say that the chance of obtaining false positive gene regulatory networks in this study would not be too much.

In conclusion, we have identified gene regulatory networks to elucidate the differences between GRs and PRs to ICSs in patients

\section{REFERENCES}

1. Goleva E, Jackson LP, Gleason M, Leung DY. Usefulness of PBMCs to predict clinical response to corticosteroids in asthmatic patients. J Allergy Clin Immunol. (2012) 129:687-93.e681. doi: 10.1016/j.jaci.2011.12.001

2. Sonawane AR, Weiss ST, Glass K, Sharma A. Network medicine in the age of biomedical big data. Front Genet. (2019) 10:294. doi: 10.3389 /fgene.2019.00294

3. Roy S, Bhattacharyya DK, Kalita JK. Reconstruction of gene co-expression network from microarray data using local expression patterns. $B M C$ Bioinformatics. (2014) 15 (Suppl. 7):S10. doi: 10.1186/1471-2105-15-S7-S10 with asthma. We identified the top-10 TFs showing different connections between GRs and PRs and found that these top-10 TFs and their differentially-connected genes were significantly enriched in distinct biological pathways, such as TGF- $\beta$ signaling, cell cycle, and IL-4 and IL-13 signaling pathways. TFs and biological pathways that were identified in this study may be potential targets to overcome insensitivity to corticosteroids in patients with asthma.

\section{CODE AVAILABILITY}

Codes generated during the current study are available upon reasonable request.

\section{DATA AVAILABILITY STATEMENT}

Immediately following publication, individual participant data that underlie the results reported in this article will be able to be shared after de-identification with researchers who will provide a methodologically sound proposal. Proposals should be directed to guinea71@snu.ac.kr.

\section{ETHICS STATEMENT}

This study was approved by the Institutional Review Board of the corresponding institution (H-1408-051-601 and 2019AN0240) and informed consent was obtained from all study participants. The patients/participants provided their written informed consent to participate in this study.

\section{AUTHOR CONTRIBUTIONS}

B-KK, H-SL, S-YL, and H-WP were involved in study conception/design. B-KK, S-YL, and H-WP were involved in data acquisition. All authors were involved in data analysis and/or interpretation, involved in writing/critical review of draft versions of this manuscript and all approved the final version for submission for publication.

\section{FUNDING}

This research was supported by a grant from the Korea University Anam Hospital, Seoul, Republic of Korea (Grant No. K1922911 and O2000691). 
7. Glass K, Huttenhower C, Quackenbush J, Yuan GC. Passing messages between biological networks to refine predicted interactions. PLOS ONE. (2013) 8:e64832. doi: 10.1371/journal.pone.0064832

8. Global Initiative for Asthma. Global Strategy for Asthma Management and Prevention, 2020. Global Initiative for Asthma (2020). Available online at: https://ginasthma.org/gina-reports/

9. Schlauch D, Paulson JN, Young A, Glass K, Quackenbush J. Estimating gene regulatory networks with pandaR. Bioinformatics. (2017) 33:2232-4. doi: 10.1093/bioinformatics/btx139

10. Han H, Cho JW, Lee S, Yun A, Kim H, Bae D, et al. TRRUST v2: an expanded reference database of human and mouse transcriptional regulatory interactions. Nucleic Acids Res. (2018) 46:D380-d386. doi: 10.1093/nar/gkx1013

11. Vargas AJ, Quackenbush J, Glass K. Diet-induced weight loss leads to a switch in gene regulatory network control in the rectal mucosa. Genomics. (2016) 108:126-33. doi: 10.1016/j.ygeno.2016.08.001

12. Reimand J, Arak T, Adler P, Kolberg L, Reisberg S, Peterson H, et al. g:Profilera web server for functional interpretation of gene lists (2016 update). Nucleic Acids Res. (2016) 44:W83-9. doi: 10.1093/nar/gkw199

13. Chowdhury S, Sarkar RR. Comparison of human cell signaling pathway databases-evolution, drawbacks and challenges. Database. (2015) 2015:bau126. doi: 10.1093/database/bau126

14. Piek E, Heldin $\mathrm{CH}$, Ten Dijke P. Specificity, diversity, and regulation in TGF-beta superfamily signaling. FASEB J. (1999) 13:2105-24. doi: 10.1096/fasebj.13.15.2105

15. Massagué J, Seoane J, Wotton D. Smad transcription factors. Genes Dev. (2005) 19:2783-810. doi: 10.1101/gad.1350705

16. Keenan CR, Mok JS, Harris T, Xia Y, Salem S, Stewart AG. Bronchial epithelial cells are rendered insensitive to glucocorticoid transactivation by transforming growth factor- $\beta 1$. Respir Res. (2014) 15:55. doi: 10.1186/1465-9921-15-55

17. Li M, Keenan CR, Lopez-Campos G, Mangum JE, Chen Q, Prodanovic $\mathrm{D}$, et al. A non-canonical pathway with potential for safer modulation of transforming growth factor- $\beta 1$ in steroid-resistant airway diseases. iScience. (2019) 12:232-46. doi: 10.1016/j.isci.2019.01.023

18. Li MO, Wan YY, Sanjabi S, Robertson AK, Flavell RA. Transforming growth factor-beta regulation of immune responses. Annu Rev Immunol. (2006) 24:99-146. doi: 10.1146/annurev.immunol.24.021605.090737

19. Marie JC, Liggitt D, Rudensky AY. Cellular mechanisms of fatal earlyonset autoimmunity in mice with the $\mathrm{T}$ cell-specific targeting of transforming growth factor-beta receptor. Immunity. (2006) 25:441-54. doi: 10.1016/j.immuni.2006.07.012

20. Sakaguchi S, Powrie F. Emerging challenges in regulatory $\mathrm{T}$ cell function and biology. Science. (2007) 317:627-9. doi: 10.1126/science.1142331

21. Strobl H, Knapp W. TGF-betal regulation of dendritic cells. Microbes Infect. (1999) 1:1283-90. doi: 10.1016/S1286-4579(99)00256-7

22. Troncone E, Marafini I, Stolfi C, Monteleone G. Transforming growth factor$\beta 1 /$ Smad7 in intestinal immunity, inflammation, and cancer. Front Immunol. (2018) 9:1407. doi: 10.3389/fimmu.2018.01407

23. Sundqvist A, Morikawa M, Ren J, Vasilaki E, Kawasaki N, Kobayashi $\mathrm{M}$, et al. JUNB governs a feed-forward network of TGF $\beta$ signaling that aggravates breast cancer invasion. Nucleic Acids Res. (2018) 46:1180-95. doi: 10.1093/nar/gkx1190

24. Sands BE, Feagan BG, Sandborn WJ, Schreiber S, Peyrin-Biroulet L, Frédéric Colombel J, et al. Mongersen (GED-0301) for active crohn's disease: results of a phase 3 study. Am J Gastroenterol. (2020) 115:738-45. doi: 10.14309/ajg.0000000000000493
25. Adcock IM, Lane SJ. Corticosteroid-insensitive asthma: molecular mechanisms. J Endocrinol. (2003) 178:347-55. doi: 10.1677/joe.0.1780347

26. Bruhn S, Katzenellenbogen M, Gustafsson M, Krönke A, Sönnichsen B, Zhang $\mathrm{H}$, et al. Combining gene expression microarray- and cluster analysis with sequence-based predictions to identify regulators of IL-13 in allergy. Cytokine. (2012) 60:736-40. doi: 10.1016/j.cyto.2012.08.009

27. Leung DY, Martin RJ, Szefler SJ, Sher ER, Ying S, Kay AB, et al. Dysregulation of interleukin 4, interleukin 5, and interferon gamma gene expression in steroid-resistant asthma. J Exp Med. (1995) 181:33-40. doi: 10.1084/jem.181.1.33

28. Harb H, Chatila TA. Mechanisms of dupilumab. Clin Exp Allergy. (2020) 50:5-14. doi: 10.1111/cea.13491

29. Bertoli C, Skotheim JM, de Bruin RA. Control of cell cycle transcription during G1 and S phases. Nat Rev Mol Cell Biol. (2013) 14:518-28. doi: $10.1038 / \mathrm{nrm} 3629$

30. Harmon JM, Norman MR, Fowlkes BJ, Thompson EB. Dexamethasone induces irreversible G1 arrest and death of a human lymphoid cell line. J Cell Physiol. (1979) 98:267-78. doi: 10.1002/jcp.1040980203

31. Hu X, Wang J, Xia Y, Simayi M, Ikramullah S, He Y, et al. Resveratrol induces cell cycle arrest and apoptosis in human eosinophils from asthmatic individuals. Mol Med Rep. (2016) 14:5231-6. doi: 10.3892/mmr.2016.5884

32. Giangrande PH, Zhu W, Schlisio S, Sun X, Mori S, Gaubatz S, et al. A role for E2F6 in distinguishing G1/S- and G2/M-specific transcription. Genes Dev. (2004) 18:2941-51. doi: 10.1101/gad.1239304

33. Desdouets C, Matesic G, Molina CA, Foulkes NS, Sassone-Corsi P, Brechot C, et al. Cell cycle regulation of cyclin A gene expression by the cyclic AMPresponsive transcription factors CREB and CREM. Mol Cell Biol. (1995) 15:3301-9. doi: 10.1128/MCB.15.6.3301

34. Shimoda M, Takahashi M, Yoshimoto T, Kono T, Ikai I, Kubo H. A homeobox protein, prox1, is involved in the differentiation, proliferation, and prognosis in hepatocellular carcinoma. Clin Cancer Res. (2006) 12 (20 Pt. 1), 6005-11. doi: 10.1158/1078-0432.CCR-06-0712

35. Kamiya A, Kakinuma S, Onodera M, Miyajima A, Nakauchi H. Prosperorelated homeobox 1 and liver receptor homolog 1 coordinately regulate longterm proliferation of murine fetal hepatoblasts. Hepatology. (2008) 48:252-64. doi: $10.1002 /$ hep. 22303

36. Fouty B, Moss T, Solodushko V, Kraft M. Dexamethasone can stimulate G1-S phase transition in human airway fibroblasts in asthma. Eur Respir J. (2006) 27:1160-7. doi: 10.1183/09031936.06.00078605

37. Liu LD, Hsiao YC, Chen HC, Yang YW, Chang MC. Construction of gene causal regulatory networks using microarray data with the coefficient of intrinsic dependence. Bot Stud. (2019) 60:22. doi: 10.1186/s40529-01 9-0268-8

38. Altay G. Empirically determining the sample size for large-scale gene network inference algorithms. IET Syst Biol. (2012) 6:35-43. doi: 10.1049/iet-syb.2010.0091

Conflict of Interest: The authors declare that the research was conducted in the absence of any commercial or financial relationships that could be construed as a potential conflict of interest.

Copyright (c) $2021 \mathrm{Kim}$, Lee, Lee and Park. This is an open-access article distributed under the terms of the Creative Commons Attribution License (CC BY). The use, distribution or reproduction in other forums is permitted, provided the original author(s) and the copyright owner(s) are credited and that the original publication in this journal is cited, in accordance with accepted academic practice. No use, distribution or reproduction is permitted which does not comply with these terms. 